\title{
Conformational substates of ferricytochrome $c$ revealed by combined optical absorption and electronic circular dichroism spectroscopy at cryogenic temperature
}

\author{
Alessandro Spilotros, Matteo Levantino, Antonio Cupane* \\ Department of Physical and Astronomical Sciences, University of Palermo, Via Archirafi 36, I-90123 Palermo, Italy
}

\section{A R T I C L E I N F O}

Article history:

Received 11 November 2009

Accepted 2 December 2009

Available online 6 December 2009

\section{Keywords}

Conformational substates

Energy landscape

Charge transfer transitions

Protein dynamics

\begin{abstract}
A B S T R A C T
We have investigated the heterogeneity of the Fe(III)-Met80 linkage of horse heart ferricytochrome $c$ by probing the $695 \mathrm{~nm}$ charge transfer band with absorption and electronic circular dichroism (ECD) spectroscopy. In order to verify the connection between conformational substates of the Fe(III)-Met80 linkage and the $695 \mathrm{~nm}$ band spectral heterogeneity, we have performed experiments as a function of $\mathrm{pH}$ (neutral and acidic) and temperature (room and $20 \mathrm{~K}$ ). At room temperature, the ECD spectrum is blue shifted with respect to the absorption one; the shift is more pronounced at acidic $\mathrm{pH}$ and is compatible with the presence of sub-bands. ECD measurements at $20 \mathrm{~K}$ highlighted the heterogeneous nature of the $695 \mathrm{~nm}$ band and provided direct experimental evidence for the presence of sub-bands. Indeed, while the absorption spectra remained deceivingly unstructured, the ECD spectra showed well resolved peaks and shoulders. A consistent fit of the $20 \mathrm{~K}$ absorption and ECD spectra showed that five Gaussians (each centered at the same frequency in the absorption and ECD spectrum) are able to reproduce the observed lineshapes. A careful analysis of frequency shifts and intensity ratios of these sub-bands enabled us to identify at least three distinct sub-bands arising from taxonomic conformational substates of the Fe(III)-Met80 linkage. In view of the major influence of the Fe(III)-Met80 linkage on the redox potential of ferricytochrome $c$, we speculate that these spectrally distinguishable substates may have different functional roles.
\end{abstract}

(C) 2009 Elsevier B.V. All rights reserved.

\section{Introduction}

The three dimensional native structure of proteins is characterized by a highly complex energy landscape in which the global minimum (corresponding to the native conformation) is made up of a large number of quasi-isoenergetic minima (corresponding to the so-called conformational substates). A hierarchical organization of the landscape has been suggested [1,2], whereby a small number of substates (the taxonomic substates) is, in turn, structured in a large number of higher tier(s) substates (the statistical substates). From the spectroscopic point of view, the statistical substates contribute to the rather large spectral Gaussian widths of protein absorption bands [3-5], while taxonomic substates are responsible for their sub-bands structure, like e.g. in the case of the near infrared charge transfer bands of deoxyhemoglobin, deoxymyoglobin and superoxide dismutase [6,7], and of the infrared stretching band of $\mathrm{CO}$ bound to myoglobin $[2,8,9]$. Protein conformational substates have substantial functional relevance, since they are responsible for the heterogeneity observed in many dynamical processes like the binding of ligands to

\footnotetext{
* Corresponding author. Dipartimento di Scienze Fisiche ed Astronomiche, Università degli Studi di Palermo, Via Archirafi 36, I-90123 Palermo, Italy. Tel.: + 39 0916234221 ; fax: + 390916162461.

E-mail address: cupane@fisica.unipa.it (A. Cupane).
}

the active site; a role of conformational substates in protein folding/ unfolding processes has also been suggested [10-12].

Cytochrome $c$ is a small globular protein with a molecular weight of about $12.4 \mathrm{kDa}$. It contains a single heme group whose central iron atom is coordinated to a histidine residue (His18, proximal side) and a methionine residue (Met80, distal side), besides the four pyrrole nitrogens of the porphyrin. Its main physiological role is to mediate the electron transfer from cytochrome $c$ reductase to cytochrome $c$ oxidase. The absorption spectrum of cytochrome $c$ in the ferric state is characterized by the well known B (Soret) and Q bands and by a weaker charge transfer (CT) band in the near infrared at about $695 \mathrm{~nm}$ [13]. This CT band had initially been assigned to an $\mathrm{A}_{2 \mathrm{u}}$ (porphyrin) $\rightarrow \mathrm{d}_{\mathrm{z}}^{2}\left(\mathrm{Fe}^{3+}\right)$ transition involving a molecular orbital of the porphyrin ring and an atomic orbital of the iron [14]. However, this band is absent in low-spin ferric forms of cytochrome $c$ whenever the Met80 residue is replaced by a different ligand $[15,16]$. This observation indicated that the $695 \mathrm{~nm}$ band should be assigned to a $\mathrm{S}(\mathrm{Met} 80) \rightarrow \mathrm{Fe}^{3+}$ transition involving atomic orbitals of the methionine sulfur and the iron ion. Indeed, the dependence of this band on the axial iron-sulfur linkage has made the band a frequently used tool to monitor the presence of intermediates in the folding/unfolding transition of ferricytochrome $c$ [17-23]. Structural heterogeneity of the heme pocket is expected to result in a spectral heterogeneity of the $695 \mathrm{~nm}$ band. Schweitzer-Stenner et al. have measured the absorption and electronic circular dichroism (ECD) spectra of ferricytochrome $c$ in the 283-333 K temperature interval 
[22]. Their data show that the absorption band at $695 \mathrm{~nm}$ is shifted with respect to the corresponding ECD band, both bands being highly asymmetric. The authors interpreted this finding as an evidence of the presence of taxonomic conformational substates and proposed the existence of at least three sub-bands (S2, S3, and S4 in their terminology). A frequency shift between absorption and ECD can be attributed to the heterogeneous nature of the spectra; indeed, if the spectra are heterogeneous and if the underlying sub-bands have different rotational and oscillator strengths, the frequency shift can be easily rationalized. A similar argument has been already used by Schweitzer-Stenner et al. also in the case of the Soret band of several myoglobin derivatives [24], although in that case the origin of spectral heterogeneity is not the presence of conformational substates, but an $x-y$ splitting due to symmetry lowering distortions. Although appealing, such evidences for sub-bands remain indirect since no clear peaks or shoulders could be resolved in the spectra close to room temperature, especially in the ECD ones. This consideration motivated the present study: in order to resolve the spectral heterogeneity of the $695 \mathrm{~nm}$ band in a clear-cut way, we have performed absorption and ECD experiments at $290 \mathrm{~K}$ and $20 \mathrm{~K}$, exploiting the widely known fact that optical absorption bands of proteins become narrower on lowering the temperature [3,25-27].

\section{Materials and methods}

Horse heart cytochrome $c$ was obtained from Sigma-Aldrich. To ensure full oxidation of the iron, a 4-fold molar excess of potassium ferricyanide was added and subsequently removed by prolonged dialysis. The protein solution was mixed with phosphate buffer and glycerol to obtain a sample of $1 \mathrm{mM}$ ferricytochrome $c$ in $65 \%$ ( $\mathrm{vol} / \mathrm{vol}$ ) glycerol/water and $0.1 \mathrm{M}$ phosphate buffer. The sample, contained in a $1 \mathrm{~cm}$ path length PMMA cuvette, remained homogeneous and transparent upon lowering the temperature down to $20 \mathrm{~K}$. Measurements have been performed at two different $\mathrm{pH}$ values, 4 and 7, in order to modulate the spectral heterogeneity while preserving intact functional and spectral properties of the protein $[17,28]$.

Absorption spectra were obtained with a Jasco V-570 spectrophotometer operated with a scan speed of $40 \mathrm{~nm} / \mathrm{min}, 1 \mathrm{~nm}$ bandwidth, and $1 \mathrm{~s}$ response time. The experimental setup for the cryogenic temperature optical absorption measurements has been described in previous publications [27,29]. For ECD measurements at cryogenic temperatures a Jasco J-715 spectropolarimeter was equipped with a home-built apparatus comprising an Oxford Optistat cryostat and an Oxford ITC 503 temperature controller; spectral parameters were as follows: scan speed $=50 \mathrm{~nm} / \mathrm{min}$, bandwidth $=2 \mathrm{~nm}$, response time $=1 \mathrm{~s} ; 20$ accumulations resulted in spectra having good signal-to-noise ratio. The frequency calibration of the spectrophotometer and spectropolarimeter were checked by measuring the absorption spectrum of a neodymium glass and the ECD spectrum of a camphor standard, respectively. We estimate that both instruments are calibrated within $20 \mathrm{~cm}^{-1}$.

At each temperature, the absorption and ECD spectra of a $65 \%$ ( $\mathrm{vol} / \mathrm{vol}$ ) glycerol/water mixture containing $0.1 \mathrm{M}$ phosphate buffer were measured and subtracted from the corresponding spectra of the protein containing sample. Control experiments made at room temperature showed that, at both $\mathrm{pH}$ values, the presence of glycerol does not introduce any relevant spectral alteration in both the absorption and ECD spectral profiles.

\section{Results and discussions}

Fig. 1 reports the absorption and ECD bands at around $695 \mathrm{~nm}$ $\left(\sim 14,400 \mathrm{~cm}^{-1}\right)$ measured at room temperature and normalized with respect to their peaks. Since both bands lie on the tail of a much more intense band, a suitable baseline has been subtracted from each spectrum: while a cubic baseline has been subtracted from the absorption spectra, a linear baseline was enough in the case of the ECD ones (see panels $c$ and $f$ of Fig. 2 below). The peak of the ECD band is blue shifted with respect to the absorption one in the ferricytochrome $c$ sample at pH 7 (Fig. 1a,b); this effect is even larger in an analogous sample at pH 4 (Fig. 1c,d). A frequency shift between an absorption band and its corresponding ECD one can be interpreted as an evidence of spectral heterogeneity as already mentioned in the Introduction. To further investigate such heterogeneity we have performed measurements at cryogenic temperatures. Fig. 2 shows the absorption spectra at 290 and $20 \mathrm{~K}$ of ferricytochrome $c$ at pH 7 (panel a) and pH 4 (panel b); the baseline subtracted from the spectrum measured at $20 \mathrm{~K}(\mathrm{pH} 4)$ is depicted in panel c. The low temperature absorption spectrum, although being, as expected, more intense and narrower, remains deceivingly
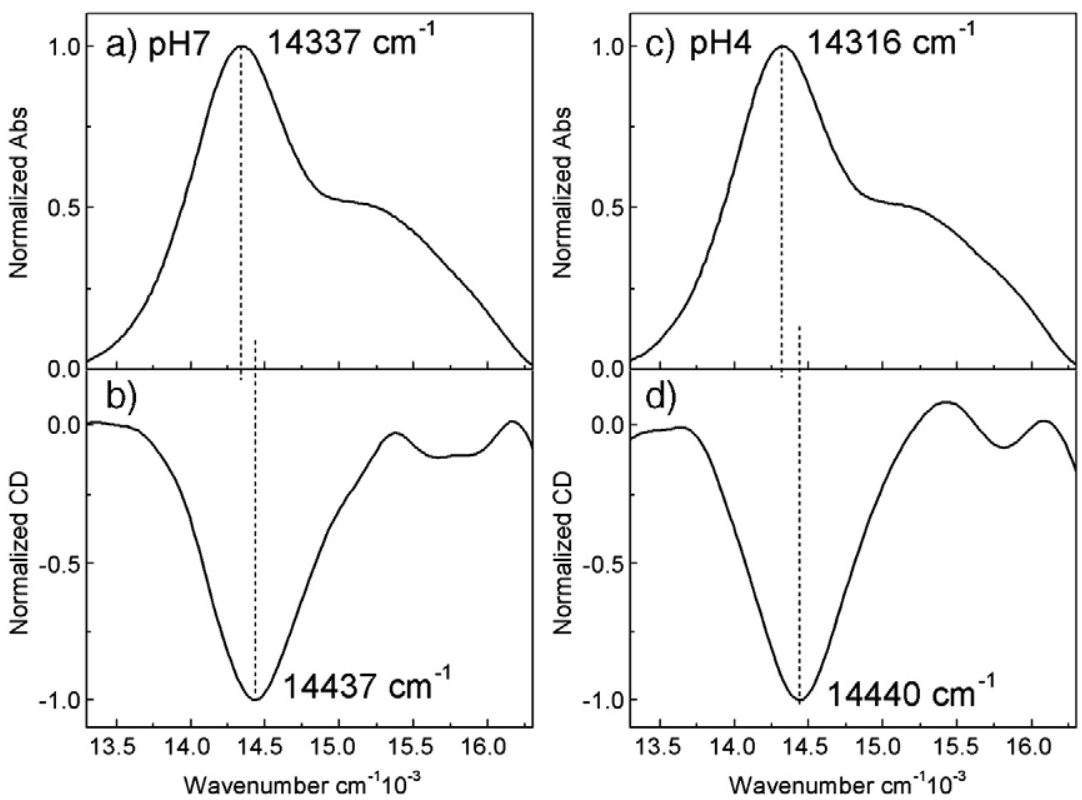

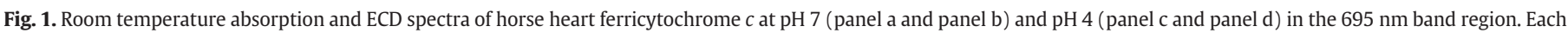
spectrum has been normalized with respect to its maximum after subtraction of a suitable baseline (see text). The dashed lines mark the position of the respective main peaks. 

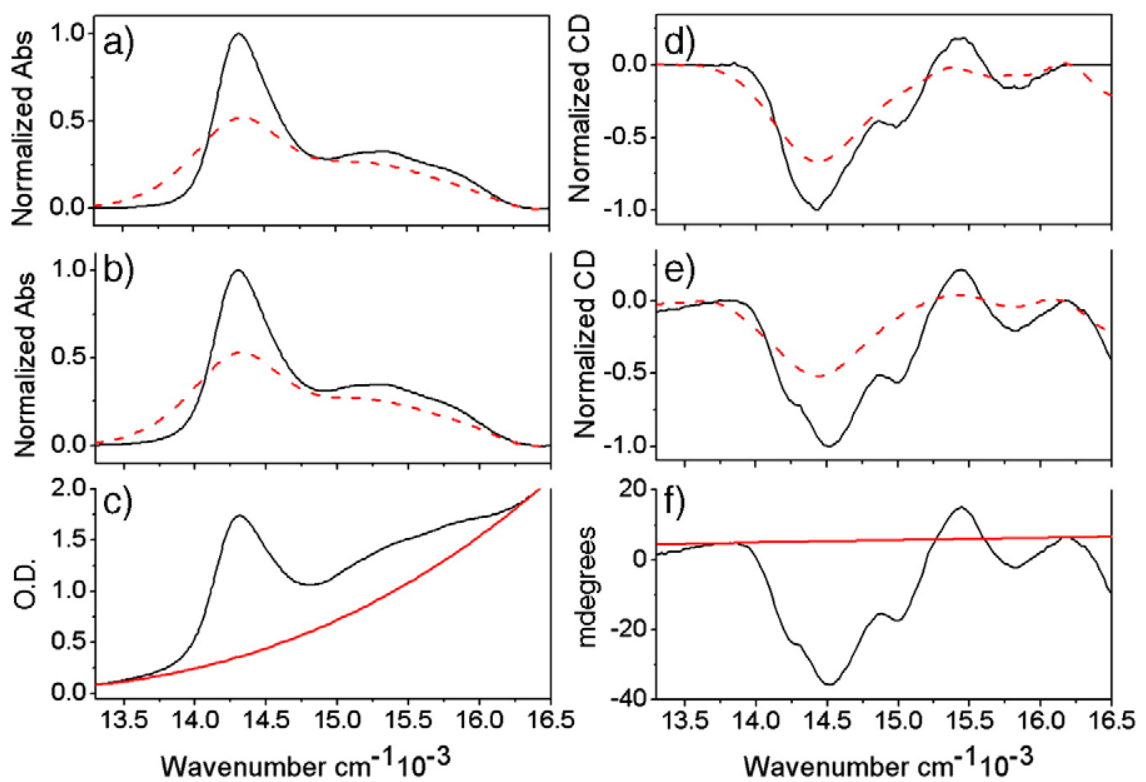

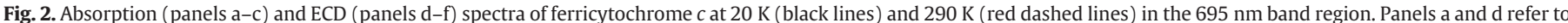

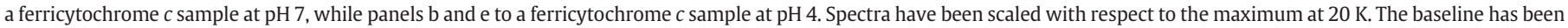
taken into account with a cubic polynomial in the case of absorption spectra (panel c) and with a linear polynomial in the case of ECD ones (panel f).

unstructured and gives no direct evidence for the presence of sub-bands. We note that the clear asymmetry of the $\sim 14,250 \mathrm{~cm}^{-1}$ absorption peak is not a proof of the presence of sub-bands since this could be related to the coupling of the underlying electronic transition with low frequency vibrational modes [26,29]. Following the idea of Schweitzer-Stenner et al. $[22,23,28,30]$, we complemented the absorption data with ECD data on the very same samples. The ECD spectrum of ferricytochrome $c$ at $20 \mathrm{~K}$ and $\mathrm{pH} 7$ (Fig. 2d) shows an asymmetric peak at $\sim 14,500 \mathrm{~cm}^{-1}$ and a clearly resolved peak at $\sim 15,000 \mathrm{~cm}^{-1}$; the high frequency wing of the spectrum is characterized by a positive peak $\mathrm{a} \sim 15,400 \mathrm{~cm}^{-1}$ and negative one at $\sim 15,800 \mathrm{~cm}^{-1}$. The residual marked asymmetry of the peak at $14,500 \mathrm{~cm}^{-1}$ seems to suggest the presence of further subbands. Indeed, the ECD spectrum of the sample at pH 4 (Fig. 2e) gives direct evidence of this hypothesis. This spectrum is very well resolved: it shows a shoulder at $\sim 14,250 \mathrm{~cm}^{-1}$, a peak at $\sim 14,500 \mathrm{~cm}^{-1}$, and a clearly resolved peak at $\sim 15,000 \mathrm{~cm}^{-1}$; as for the $\mathrm{pH} 7$ sample, the high frequency wing is characterized by peaks at approximately 15,400 and $15,800 \mathrm{~cm}^{-1}$. Note that the small band at about $13,650 \mathrm{~cm}^{-1}$ reported by Dragomir et al. [23] and named S1 by the authors, is never observed in our experiments (both in absorption and ECD) even at $20 \mathrm{~K}$.

Based on direct evidence given by the $20 \mathrm{~K} \mathrm{ECD}$ spectrum at $\mathrm{pH} 4$, we conclude that the $695 \mathrm{~nm}$ absorption and ECD bands of ferricytochrome $c$ are composed of several spectrally distinguishable sub-bands having different oscillator and rotational strengths. These spectroscopically distinct sub-bands likely correspond to different structures, i.e. to different conformational substates. Note that this spectral heterogeneity is already evident by inspection of the raw data, and it does not depend on the details of the baseline subtraction procedure.

To obtain quantitative information on the composite structure of the $695 \mathrm{~nm}$ band, and to check the consistency of our hypotheses, we analyzed simultaneously the absorption and ECD spectra measured at $20 \mathrm{~K}$ between 13,500 and $16,500 \mathrm{~cm}^{-1}$; five different Gaussian components sharing the same peak frequencies in the absorption and ECD spectra have been used to reproduce the observed spectral profiles. Fig. 3 shows the result of the fitting procedure and demonstrates that the absorption and ECD spectra can be well fitted with a single set of five Gaussian sub-bands that are named A1 to A5 in the figure. Best fit parameters for the areas $\left(I_{k}, k=1 \ldots 5\right)$, peak frequencies $\left(\nu_{k}, k=1 \ldots 5\right)$, and widths $\left(\sigma_{k}, k=1 \ldots 5\right)$ of the five
Gaussian components are reported in Table 1. The fractional areas of the sub-bands are reported in Fig. 4.

The data in Figs. 3 and 4 and in Table 1 show that:

- The area of the sub-bands depends sizably on $\mathrm{pH}$, particularly in the case of the ECD spectrum: indeed, at $\mathrm{pH} 4$, the ratio $I_{2} / I_{1}$ is $\sim 1.37$ (i.e. the rotational strength of $\mathrm{A} 1$ is smaller than that of $\mathrm{A} 2$ ), while at $\mathrm{pH} 7$ it is $\sim 0.83$ (i.e. the rotational strength of $\mathrm{A} 1$ is larger than that of A2). This makes the ECD spectrum at pH 4 more resolved than the corresponding absorption spectrum.

- At a given $\mathrm{pH}$, the areas of the sub-bands scale differently in the absorption and ECD spectra (Fig. 4): e.g., in the case of the sample at $\mathrm{pH} 7, I_{2} / I_{1}$ is $\sim 0.45$ and $\sim 0.83$ for the absorption and ECD spectrum, respectively, while, at $\mathrm{pH} 4, I_{2} / I_{1}$ is $\sim 0.50$ for absorption and $\sim 1.37$ for ECD.

- Frequency separations between sub-bands depend weakly on $\mathrm{pH}$ and are $\sim 300 \mathrm{~cm}^{-1}\left(\nu_{2}-\nu_{1}\right), \sim 700 \mathrm{~cm}^{-1}\left(\nu_{3}-\nu_{1}\right), \sim 1100 \mathrm{~cm}^{-1}$ $\left(\nu_{4}-\nu_{1}\right)$, and $\sim 1530 \mathrm{~cm}^{-1}\left(\nu_{5}-\nu_{1}\right)$.

- Although they were left as free parameters in the fitting procedure, the widths of the sub-bands in the ECD spectrum are very similar to those of the absorption one (Table 1). A significant discrepancy is observed only in the case of the A4 sub-band (at $\sim 15,400 \mathrm{~cm}^{-1}$ ) whose width is, however, very sensitive to the details of the baseline subtraction procedure, especially for the ECD spectrum.

On the basis of our data we conclude that the spectrally distinguishable A1, A2, and A3 components are different sub-bands of the same $\mathrm{p}(\mathrm{S}) \rightarrow \mathrm{d}_{\pi}\left(\mathrm{Fe}^{3+}\right)$ transition arising from different cytochrome $c$ taxonomic conformational substates [16]. Supporting evidence for this conclusion comes from the following arguments:

1) Though it would seem possible to assign the different sub-bands to different $\mathrm{p}(\mathrm{S}) \rightarrow \mathrm{d}_{\pi}\left(\mathrm{Fe}^{3+}\right)$ transitions, the observed sub-bands frequency differences are at variance with such conclusion. In fact, the magnetic circular dichroism (MCD) data reported by McKnight et al. [16] suggest a wavenumber difference of nearly $1000 \mathrm{~cm}^{-1}$ between, e.g., $\mathrm{p}_{\mathrm{y}} \rightarrow \mathrm{d}_{\mathrm{yz}}$ and $\mathrm{p}_{\mathrm{z}} \rightarrow \mathrm{d}_{\mathrm{yz}}$, while the $\nu_{2}-\nu_{1}$ and $\nu_{3}-\nu_{1}$ frequency differences are sizably smaller than $1000 \mathrm{~cm}^{-1}$.

2) Our spectra rule out the hypothesis of a vibronic origin of these sub-bands since the $I_{2} / I_{1}$ and $I_{3} / I_{1}$ ratios scale differently in the 

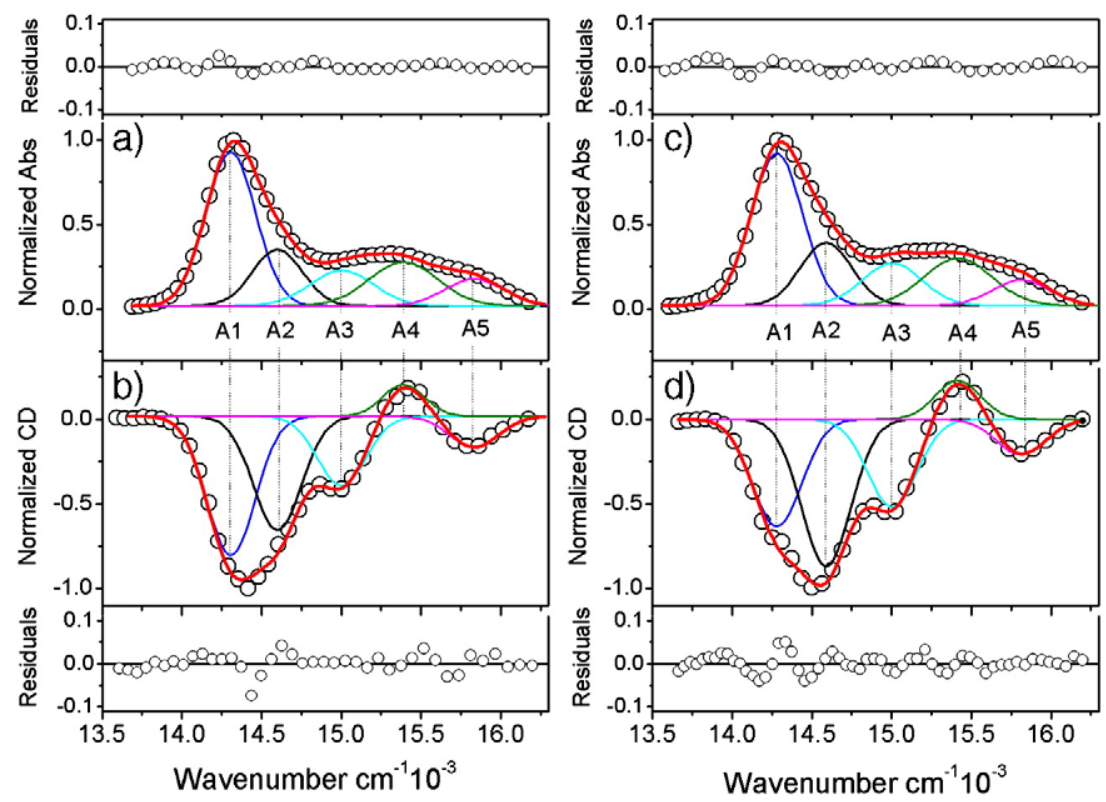

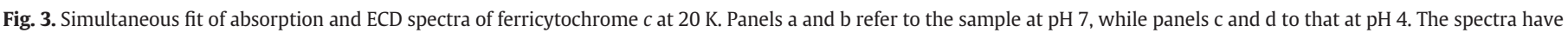

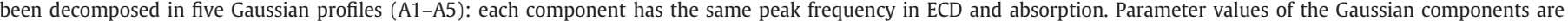
reported in Table 1 .

absorption and ECD spectra. As a matter of fact, it is possible to demonstrate that vibronic replicas in the ECD spectra are simply proportional to those in the absorption ones [31,32].

Coming to the origin of the high frequency wing centered at $\sim 15,400 \mathrm{~cm}^{1}(\sim 650 \mathrm{~nm})$ and corresponding to the A4 and A5 Gaussian components, our data do not allow us to make an unambiguous assignment since the arguments used above cannot be applied. Indeed, the $\nu_{4}-\nu_{1}$ and $\nu_{5}-\nu_{1}$ frequency separations are greater than $1000 \mathrm{~cm}^{-1}$, and indeterminations in the baseline subtraction procedure (that has a stronger influence in this frequency region) do not allow us to draw definitive conclusions on the intensity scaling. The high frequency wing could, thus, well originate also from a different $\mathrm{CT}$ transition, or from porphyrin skeletal vibrations coupled to the main CT transition [14,16,33]. As far as the first hypothesis is concerned, the most reasonable assignment, following the arguments of McKnight et al. [16], is that of a $\mathrm{p}_{\mathrm{z}}(\mathrm{S}) \rightarrow \mathrm{d}_{\mathrm{yz}}\left(\mathrm{Fe}^{3+}\right)$ transition as opposed to the $\mathrm{p}_{\mathrm{y}}(\mathrm{S}) \rightarrow \mathrm{d}_{\mathrm{yz}}\left(\mathrm{Fe}^{3+}\right)$ transition at $695 \mathrm{~nm}$. According to such assignment the low frequency transition is expected to be $z$-polarized, while the high frequency one should be $x, y$-polarized.

Table 1

Best fit parameters of the five Gaussian sub-bands (A1-A5) used to fit simultaneously the absorption and ECD spectra at $20 \mathrm{~K}$. The typical uncertainty on the best fit values for the areas $\left(I_{k}, k=1 \ldots 5\right)$ is $\leq 0.005$, while that for frequencies $\left(\nu_{k}, k=1 \ldots 5\right)$ and widths $\left(\sigma_{k}, k=1 \ldots 5\right)$ is $\leq 20 \mathrm{~cm}^{-1}$.

\begin{tabular}{|c|c|c|c|c|}
\hline & Abs pH 7 & ECD pH 7 & Abs pH 4 & $\mathrm{ECD} \mathrm{pH} 4$ \\
\hline$I_{1}$ (a.u.) & 0.410 & -0.323 & 0.425 & -0.244 \\
\hline$I_{2}$ (a.u.) & 0.185 & -0.268 & 0.211 & -0.335 \\
\hline$I_{3}$ (a.u.) & 0.159 & -0.170 & 0.178 & -0.200 \\
\hline$I_{4}$ (a.u.) & 0.196 & 0.107 & 0.210 & 0.081 \\
\hline$I_{5}$ (a.u.) & 0.129 & -0.088 & 0.129 & -0.081 \\
\hline$\nu_{1}\left(\mathrm{~cm}^{-1}\right)$ & \multicolumn{2}{|c|}{14310} & \multicolumn{2}{|c|}{14280} \\
\hline$\nu_{2}\left(\mathrm{~cm}^{-1}\right)$ & \multicolumn{2}{|c|}{14600} & \multicolumn{2}{|c|}{14590} \\
\hline$\nu_{3}\left(\mathrm{~cm}^{-1}\right)$ & \multicolumn{2}{|c|}{15000} & \multicolumn{2}{|c|}{15000} \\
\hline$\nu_{4}\left(\mathrm{~cm}^{-1}\right)$ & \multicolumn{2}{|c|}{15390} & \multicolumn{2}{|c|}{15420} \\
\hline$\nu_{5}\left(\mathrm{~cm}^{-1}\right)$ & \multicolumn{2}{|c|}{15830} & \multicolumn{2}{|c|}{15810} \\
\hline$\sigma_{1}\left(\mathrm{~cm}^{-1}\right)$ & 160 & 150 & 160 & 150 \\
\hline$\sigma_{2}\left(\mathrm{~cm}^{-1}\right)$ & 160 & 150 & 160 & 150 \\
\hline$\sigma_{3}\left(\mathrm{~cm}^{-1}\right)$ & 160 & 150 & 160 & 150 \\
\hline$\sigma_{4}\left(\mathrm{~cm}^{-1}\right)$ & 320 & 130 & 320 & 140 \\
\hline$\sigma_{5}\left(\mathrm{~cm}^{-1}\right)$ & 150 & 150 & 150 & 150 \\
\hline
\end{tabular}

Accurate low temperature polarized absorption, resonance Raman with excitation in the near infrared, and spectral hole burning experiments on the $695 \mathrm{~nm}$ band would be needed in order to make an unambiguous assignment of the high frequency side-bands.

In the absence of direct structural evidence, we can only speculate about the structural origin of the A1, A2, and A3 sub-bands. In view of the charge transfer character of the associated transition, the ironmethionine linkage must obviously be involved. Different orientations of the S-Fe bond might be a plausible origin. However, the $\mathrm{pH}$ dependence of sub-bands intensity ratios suggests that they may be related to substates having different protonation states of residues pointing inside the heme pocket (close to the iron-methionine linkage). Finally, since the spectral heterogeneity is more pronounced for ECD than for absorption, a difference in the overall conformation and chirality of the heme pocket is also expected. In view of the major influence of the $\mathrm{Fe}^{3+}$-Met80 linkage on the redox potential of the protein [34], we speculate that the above spectrally distinguishable substates may as well have different functional roles. Such hypothesis should be tested and taken into account also in future studies employing the $695 \mathrm{~nm}$ band as a conformational marker sensitive to the folding/unfolding of cytochrome $c$.
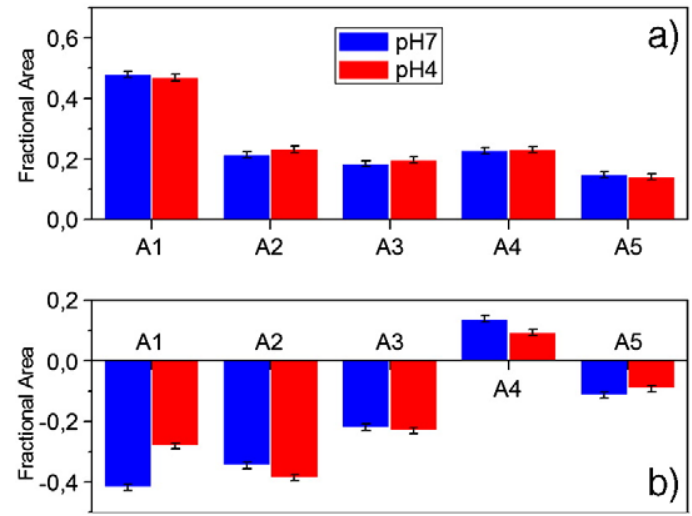

Fig. 4. Fractional areas of the Gaussian sub-bands for the absorption (panel a) and ECD (panel b) spectra with corresponding error bars. Fractional areas have been calculated as the ratio between the area of a single sub-band and the total area of the spectrum. 


\section{Conclusions}

The combined use of low temperature optical absorption and ECD spectroscopies unambiguously shows that the ferricytochrome $c$ spectrum in the $13,500-16,500 \mathrm{~cm}^{-1}(610-740 \mathrm{~nm})$ region can be decomposed in five sub-bands that we label A1-A5. Detailed inspection of frequency shifts and intensity ratios enabled us to identify components $A 1, A 2$ and $A 3$ as sub-bands of the same $p_{y}(S) \rightarrow d_{y z}\left(F^{3+}\right)$ electronic transition, arising from different cytochrome $c$ taxonomic substates. For A4 and A5 the assignment is more ambiguous and needs further experimental investigations with different spectroscopic techniques.

The present results highlight the potentialities of combining low temperature absorption and ECD for the resolution of highly heterogeneous protein spectra.

\section{Acknowledgments}

We thank Prof. M. Leone for providing very helpful discussions, Dr. E. Vitrano for generous help in developing the system for ECD measurements at cryogenic temperatures, and Mr. G. Napoli of the cryogenic laboratory for technical help.

\section{References}

[1] H. Frauenfelder, F. Parak, R.D. Young, Conformational substates in proteins, Annu. Rev. Biophys. Biophys. Chem. 17 (1988) 451-479.

[2] H. Frauenfelder, B.H. McMahon, R.H. Austin, K. Chu, J.T. Groves, The role of structure, energy landscape, dynamics, and allostery in the enzymatic function of myoglobin, Proc. Natl. Acad. Sci. U.S.A. 98 (2001) 2370-2374.

[3] A. Cupane, M. Leone, E. Vitrano, L. Cordone, Low temperature optical absorption spectroscopy: an approach to the study of stereodynamic properties of hemeproteins, Eur. Biophys. J. 23 (1995) 385-398.

[4] M. Levantino, A. Cupane, L. Zimanyi, Quaternary structure dependence of kinetic hole burning and conformational substates interconversion in hemoglobin, Biochemistry 42 (2003) 4499-4505.

[5] M. Levantino, A. Cupane, L. Zimanyi, P. Ormos, Different relaxations in myoglobin after photolysis, Proc. Natl. Acad. Sci. U S A 101 (2004) 14402-14407.

[6] A. Cupane, M. Leone, V. Militello, M.E. Stroppolo, F. Polticelli, A. Desideri, Lowtemperature optical spectroscopy of native and azide-reacted bovine $\mathrm{Cu}, \mathrm{Zn}$ superoxide dismutase. A structural dynamics study, Biochemistry 33 (1994) 15103-15109.

[7] H. Gilch, R. SchweitzerStenner, W. Dreybrodt, M. Leone, A. Cupane, L. Cordone, Conformational substates of the Fe2+-His F8 linkage in deoxymyoglobin and hemoglobin probed in parallel by the Raman band of the Fe-His stretching vibration and the near infrared absorption band III, Int. J. Quantum Chem. 59 (1996) 301-313.

[8] P. Ormos, A. Ansari, D. Braunstein, B.R. Cowen, H. Frauenfelder, M.K. Hong I.E. Iben, T.B. Sauke, P.J. Steinbach, R.D. Young, Inhomogeneous broadening in spectral bands of carbonmonoxymyoglobin. The connection between spectral and functional heterogeneity, Biophys. J. 57 (1990) 191-199.

[9] J.D. Muller, B.H. McMahon, E.Y. Chien, S.G. Sligar, G.U. Nienhaus, Connection between the taxonomic substates and protonation of histidines 64 and 97 in carbonmonoxy myoglobin, Biophys. J. 77 (1999) 1036-1051.

[10] H. Frauenfelder, S.G. Sligar, P.G. Wolynes, The energy landscapes and motions of proteins, Science 254 (1991) 1598-1603.

[11] D.C. Sullivan, I.D. Kuntz, Conformation spaces of proteins, Proteins 42 (2001) 495-511.

[12] H. Frauenfelder, P.W. Fenimore, G. Chen, B.H. McMahon, Protein folding is slaved to solvent motions, Proc. Natl. Acad. Sci. U.S.A. 103 (2006) 15469-15472.
[13] A. Schejter, P. George, The 695-millimicron band of ferricytochrome $c$ and its relationship to protein conformation, Biochemistry 3 (1964) 1045-1049.

[14] W.A. Eaton, R.M. Hochstrasser, Electronic spectrum of single crystals of ferricytochrome-c, J. Chem. Phys. 46 (1967) 2533-2539.

[15] J.J. Rux, J.H. Dawson, Magnetic circular dichroism spectroscopy as a probe of axial heme ligand replacement in semisynthetic mutants of cytochrome $c$, FEBS Lett. 290 (1991) 49-51.

[16] J.McKnight, M.R Cheesman, A.J. Thomson, J.S. Miles, A.W Munro, Identification of charge-transfer transitions in the optical-spectrum of low-spin ferric cytochromeP-450 Bacillus-megaterium, Eur. J. Biochem. 213 (1993) 683-687.

[17] C. Greenwood, M.T. Wilson, Studies on ferricytochrome c. I. Effect of pH, ionic strength and protein denaturants on the spectra of ferricytochrome $c$, Eur. J. Biochem. 22 (1971) 5-10

[18] T.A. Moore, C. Greenwood, A method for investigating the effect of temperature on the $695 \mathrm{~nm}$ band of insoluble cytochrome c, Biochem. J. 149 (1975) 169-177.

[19] Y.P. Myer, Ferricytochrome $c$. Refolding and the methionine 80-sulfur-iron linkage, J. Biol. Chem. 259 (1984) 6127-6133.

[20] L.L. Pearce, A.L. Gartner, M. Smith, A.G. Mauk, Mutation-induced perturbation of the cytochrome $c$ alkaline transition, Biochemistry 28 (1989) 3152-3156.

[21] K.M. Pryse, T.G. Bruckman, B.W. Maxfield, E.L. Elson, Kinetics and mechanism of the folding of cytochrome $c$, Biochemistry 31 (1992) 5127-5136.

[22] R. Schweitzer-Stenner, R. Shah, A. Hagarman, I. Dragomir, Conformational substates of horse heart cytochrome $c$ exhibit different thermal unfolding of the heme cavity, J. Phys. Chem. B 111 (2007) 9603-9607.

[23] I. Dragomir, A. Hagarman, C. Wallace, R. Schweitzer-Stenner, Optical band splitting and electronic perturbations of the heme chromophore in cytochrome $c$ at room temperature probed by visible electronic circular dichroism spectroscopy, Biophys. J. 92 (2007) 989-998.

[24] R. Schweitzer-Stenner, J.P. Gorden, A. Hagarman, Asymmetric band profile of the Soret band of deoxymyoglobin is caused by electronic and vibronic perturbations of the heme group rather than by a doming deformation, J. Chem. Phys. 127 (2007) 135103

[25] L. Cordone, A. Cupane, M. Leone, E. Vitrano, Thermal-behavior of the 760-Nm absorption-band in photodissociated sperm whale carbonmonoxymyoglobin at cryogenic temperature-dependence on external medium, Biopolymers 29 (1990) 639-643.

[26] A. Cupane, M. Leone, E. Unger, C. Lemke, M. Beck, W. Dreybrodt, R. SchweitzerStenner, Dynamics of various metal-octaethylporphyrins in solution studied by resonance Raman and low-temperature optical absorption spectroscopies. Role of the central metal, J. Phys. Chem. B 102 (1998) 6612-6620.

[27] M. Levantino, Q. Huang, A. Cupane, M. Laberge, A. Hagarman, R. SchweitzerStenner, The importance of vibronic perturbations in ferrocytochrome $c$ spectra: a reevaluation of spectral properties based on low-temperature optical absorption, resonance Raman, and molecular-dynamics simulations, J. Chem. Phys. 123 (2005) 054508.

28] D. Verbaro, A. Hagarman, J. Soffer, R. Schweitzer-Stenner, The pH-dependence of the $695 \mathrm{~nm}$ charge transfer band reveals the population of an intermediate state of the alkaline transition of ferricytochrome $c$ at low ion concentrations, Biochemistry 48 (2009) 2990-2996.

[29] M.G. Santangelo, M. Levantino, E. Vitrano, A. Cupane, Ferricytochrome c encapsulated in silica hydrogels: correlation between active site dynamics and solvent structure, Biophys. Chem. 103 (2003) 67-75.

[30] R. Shah, R. Schweitzer-Stenner, Structural changes of horse heart ferricytochrome $c$ induced by changes of ionic strength and anion binding, Biochemistry 47 (2008) 5250-5257.

[31] W. Moffit, A. Moscowitz, Optical activity in absorbing media, J. Chem. Phys. 30 (1959) 648-661.

[32] D.J. Caldwell, Vibronic theory of circular dichroism, J. Chem. Phys. 51 (1969) 984-988.

[33] S.Z. Hu, I.K. Morris, J.P. Singh, K.M. Smith, T.G. Spiro, Complete assignment of cytochrome- $c$ resonance Raman-spectra via enzymatic reconstitution with isotopically labeled hemes, J. Am. Chem. Soc. 115 (1993) 12446-12458.

[34] C.J. Wallace, A.E. Proudfoot, On the relationship between oxidation-reduction potential and biological activity in cytochrome $c$ analogues. Results from four novel two-fragment complexes, Biochem. J. 245 (1987) 773-779. 